\title{
Rapid breath analysis for acute respiratory distress syndrome diagnostics using a portable two-dimensional gas chromatography device
}

\author{
Menglian Zhou ${ }^{1} \cdot$ Ruchi Sharma ${ }^{1} \cdot$ Hongbo Zhu $^{1} \cdot$ Ziqi Li $^{1} \cdot$ Jiliang $\mathrm{Li}^{1} \cdot$ Shiyu Wang ${ }^{1} \cdot$ Erin Bisco ${ }^{2,3}$ - Justin Massey ${ }^{2,3}$. \\ Amanda Pennington ${ }^{2,3}$. Michael Sjoding ${ }^{3,4}$ • Robert P. Dickson ${ }^{3,4}$ - Pauline Park ${ }^{3,5}$ - Robert Hyzy ${ }^{3,4}$. \\ Lena Napolitano ${ }^{3,5} \cdot$ Christopher E. Gillies $^{2,3} \cdot$ Kevin R. Ward ${ }^{2,3} \cdot$ Xudong Fan ${ }^{1,3}$
}

Received: 2 May 2019 / Revised: 24 June 2019 / Accepted: 5 July 2019 / Published online: 1 August 2019

(C) Springer-Verlag GmbH Germany, part of Springer Nature 2019

\begin{abstract}
Acute respiratory distress syndrome (ARDS) is the most severe form of acute lung injury, responsible for high mortality and long-term morbidity. As a dynamic syndrome with multiple etiologies, its timely diagnosis is difficult as is tracking the course of the syndrome. Therefore, there is a significant need for early, rapid detection and diagnosis as well as clinical trajectory monitoring of ARDS. Here, we report our work on using human breath to differentiate ARDS and non-ARDS causes of respiratory failure. A fully automated portable 2-dimensional gas chromatography device with high peak capacity (>200 at the resolution of 1), high sensitivity (sub-ppb), and rapid analysis capability $(\sim 30 \mathrm{~min})$ was designed and made in-house for on-site analysis of patients' breath. A total of 85 breath samples from 48 ARDS patients and controls were collected. Ninety-seven elution peaks were separated and detected in $13 \mathrm{~min}$. An algorithm based on machine learning, principal component analysis (PCA), and linear discriminant analysis (LDA) was developed. As compared to the adjudications done by physicians based on the Berlin criteria, our device and algorithm achieved an overall accuracy of $87.1 \%$ with $94.1 \%$ positive predictive value and $82.4 \%$ negative predictive value. The high overall accuracy and high positive predicative value suggest that the breath analysis method can accurately diagnose ARDS. The ability to continuously and non-invasively monitor exhaled breath for early diagnosis, disease trajectory tracking, and outcome prediction monitoring of ARDS may have a significant impact on changing practice and improving patient outcomes.
\end{abstract}

Keywords Breath analysis $\cdot$ Acute respiratory distress syndrome gas chromatography $\cdot 2 \mathrm{D}$ GC $\cdot$ Machine learning

Menglian Zhou and Ruchi Sharma contributed equally to this work.

Electronic supplementary material The online version of this article (https://doi.org/10.1007/s00216-019-02024-5) contains supplementary material, which is available to authorized users.

Kevin R. Ward

keward@med.umich.edu

Xudong Fan

xsfan@umich.edu

1 Department of Biomedical Engineering, University of Michigan, 1101 Beal Ave, Ann Arbor, MI 48109, USA

2 Department of Emergency Medicine, University of Michigan, 1500 E. Medical Center Drive, Ann Arbor, MI 48109, USA
Michigan Center for Integrative Research in Critical Care, 2800 Plymouth Rd, Ann Arbor, MI 48109, USA

4 Department of Internal Medicine: Division of Pulmonary and Critical Care, 1500 E. Medical Center Drive, Ann Arbor, MI 48109, USA

5 Department of Surgery: Section of Acute Care Surgery, 1500 E. Medical Center Drive, Ann Arbor, MI 48109, USA 


\section{Introduction}

Acute respiratory distress syndrome (ARDS) is an inflammatory condition of the lung producing severe lung damage. It is one of the most severe forms of acute lung injury and responsible for high mortality (40\%) and long-term morbidity [1-3]. An estimated 200,000 Americans develop ARDS each year, of which more than 74,000 die from the disease [1]. Patients who survive ARDS experience long-term deficits in physical and neurocognitive function $[4,5]$. Both primary hospitalizations and increased health service utilization among survivors are associated with high healthcare costs [1, 4]. For example, the average cost of an ICU (Intensive Care Unit) patient requiring mechanical ventilation ranges between $\$ 7000$ and $\$ 11,000$ per day with an incremental cost of $\$ 1000-1500$ per day for mechanical ventilation [6].

Numerous predisposing factors for ARDS have previously been identified (e.g., sepsis, aspiration, and trauma) [7]. However, our understanding of patient susceptibility to ARDS is incomplete and the disease onset is poorly predicted by current risk models. Among patients with multiple established risk factors, the majority do not develop ARDS, while a minority develop severe, life-threatening disease $[8$, 9]. The most commonly used ARDS risk model (Lung Injury Prediction Score, LIPS) has strong negative predictive value (97\%), effectively identifying patients at low risk for ARDS, but weak positive predictive value $(18 \%)[1,8]$, implying poor ability to predict disease onset. The clinical diagnosis of ARDS is established based on the radiological, physiological, and clinical criteria summarized in the Berlin definition (Table 1) [9]. However, those criteria only show a moderate correlation with real-time and post-mortem tissue pathological findings $[10,11]$ and temporally lag the acute, dynamic inflammatory processes responsible for ARDS, and thus cannot be used for early diagnosis and trajectory monitoring of ARDS. Therefore, there is a significant unmet clinical need for early, rapid detection and diagnosis as well as clinical trajectory monitoring of ARDS.
Exhaled breath condensate (non-volatile compounds) of ARDS patients have been studied actively for years to help understanding of the natural history, pathophysiology, and prognosis of ARDS $[12,13]$. For example, a novel and noninvasive sampling method using heat-moisture exchanger (HME) filter [14] was developed very recently to accurately sample the distal airspace in patients with ARDS. The HME filter is an inline hygroscopic sponge placed between the patient and the ventilator, the moisture from the patient's exhaled breath condenses on this filter. By clinical practice, this filter should be changed every few hours, then the condensed fluid can be collected from the used filter and analyzed using liquid chromatography coupled tandem mass spectrometry (LCMS). While potentially useful in ARDS diagnosis, this technology is focused on proteomic analysis of the breath condensates and requires long analysis time.

Hundreds of volatile organic compounds (VOCs) are also contained in exhaled breath. Many VOCs (such as pentane, isoprene, and ethane) are related to inflammatory processes occurring in the lungs and systemically in blood from remote organ injury [15-18]. Those and other VOCs could potentially be used as biomarkers to predict the onset and severity of certain critical lung diseases such as ARDS as well as systemic inflammation such as sepsis. They also could help guide therapy if they could be measured simultaneously and precisely in real-time [19-22]. Unlike blood-based analysis, breath is unlimited in its sampling potential and can be non-invasively and continuously collected and analyzed. Technologies designed for the real-time analysis of VOCs in a point-of-care (POC) fashion could allow for the identification of breathomic signatures that enable the early diagnosis of ARDS, stratification, and trajectory monitoring, allowing for precision treatments.

Table 2 summarizes the major technologies used in breath analysis. A more comprehensive overview of the different technologies can also be found in Saalberg et al. [35] and Cao et al. [36]. Gas chromatography in tandem with mass spectrometer (GC-MS) is the gold standard for the analysis of complex vapor mixtures such as breath samples. In

Table 1 The Berlin definition of acute respiratory distress syndrome (ARDS)

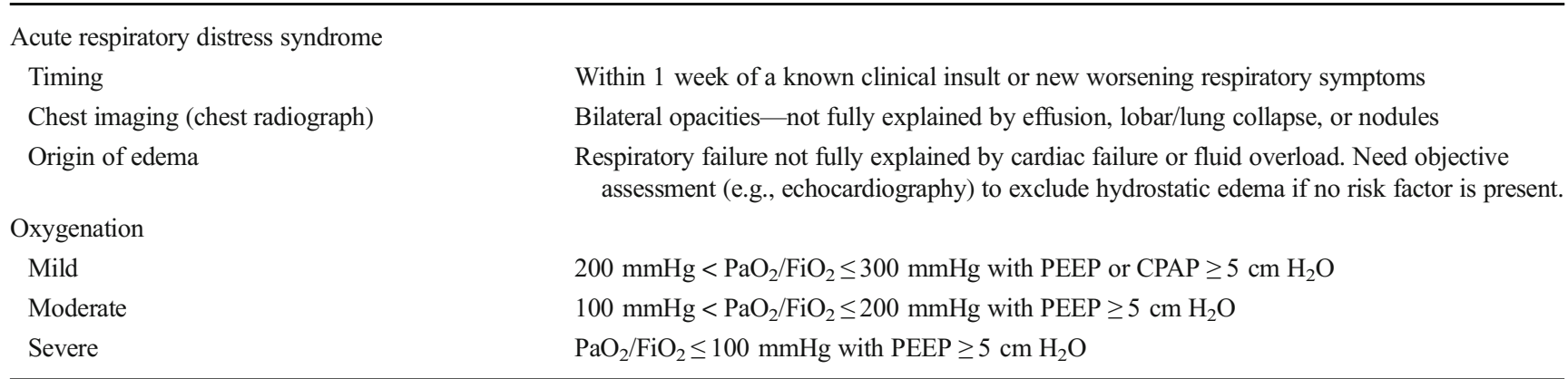

$\mathrm{CPAP}$ continuous positive airway pressure, $\mathrm{FiO}_{2}$ fraction of inspired oxygen, $\mathrm{PaO}_{2}$ partial pressure of arterial oxygen, $\mathrm{PEEP}$ positive end-expiratory pressure 
Table 2 A summary of breath analysis technologies

\begin{tabular}{|c|c|c|c|c|c|c|c|}
\hline Analysis method & Instrument & $\begin{array}{l}\text { Sampling } \\
\text { method }\end{array}$ & LOD & $\begin{array}{l}\text { Analysis } \\
\text { time }\end{array}$ & Advantages & Limitations & $\begin{array}{l}\text { Suitable } \\
\text { for POC } \\
\text { application }\end{array}$ \\
\hline $\begin{array}{l}\text { Benchtop GC [23, } \\
\text { 24] }\end{array}$ & $\begin{array}{l}\text { GC-FID/TCD }{ }^{\mathrm{a}} \text {; GC-MS; } \\
\text { GCxGC-MS; }\end{array}$ & $\begin{array}{l}\text { Sorbent trap } \\
\text { SPME }\end{array}$ & $\mathrm{ppt}^{\mathrm{f}}$ & Long & $\begin{array}{l}\text { Most widely used; High } \\
\text { separation capability; } \\
\text { compound identification } \\
\text { available }\end{array}$ & $\begin{array}{l}\text { Bulky size; heavy } \\
\text { weight; dedicated } \\
\text { personnel needed; } \\
\text { sample preparation } \\
\text { needed }\end{array}$ & No \\
\hline $\begin{array}{l}\text { Selected-ion } \\
\text { flow-tube } \\
{[25-27]}\end{array}$ & SIFT-MS & Direct input & $\begin{array}{c}\mathrm{ppb}^{\mathrm{g}} \text { to } \\
\mathrm{ppt}\end{array}$ & Real time & $\begin{array}{l}\text { Rapid analysis; high } \\
\text { sensitivity; allow } \\
\text { breath-by-breath analyses; } \\
\text { no sample preparation } \\
\text { needed }\end{array}$ & $\begin{array}{l}\text { Bulky size; heavy } \\
\text { weight; high cost; } \\
\text { dedicated personnel } \\
\text { needed }\end{array}$ & No \\
\hline $\begin{array}{l}\text { Proton transfer } \\
\text { reaction [28] }\end{array}$ & PTR-MS & Direct input & $\begin{array}{c}\text { ppb to } \\
\text { ppt }\end{array}$ & Real time & $\begin{array}{l}\text { Rapid analysis; high } \\
\text { sensitivity; allow } \\
\text { breath-by-breath analyses; } \\
\text { no sample preparation } \\
\text { needed }\end{array}$ & $\begin{array}{l}\text { Bulky size; heavy } \\
\text { weight; high cost; } \\
\text { dedicated personnel } \\
\text { needed }\end{array}$ & No \\
\hline $\begin{array}{l}\text { Ion mobility } \\
\text { spectrometry } \\
{[29-31]}\end{array}$ & FAIMS $^{\mathrm{b}}$ & Direct input & $\begin{array}{l}\mathrm{ppm}^{\mathrm{h}} \text { to } \\
\mathrm{ppb}\end{array}$ & Short & $\begin{array}{l}\text { Portable; fast; no sample } \\
\text { preparation needed }\end{array}$ & $\begin{array}{l}\text { Low separation } \\
\text { capability; } \\
\text { background VOC } \\
\text { interference }\end{array}$ & Yes \\
\hline $\begin{array}{c}\text { Electronic nose } \\
{[21,32,33]}\end{array}$ & $\begin{array}{l}\text { AuNPs }^{\mathrm{c}} ; \mathrm{CNTs}^{\mathrm{d}} ; \mathrm{CPs}^{\mathrm{e}} ; \\
\text { Colormetric; }\end{array}$ & Direct input & $\begin{array}{l}\mathrm{ppm} \text { to } \\
\mathrm{ppb}\end{array}$ & Short & $\begin{array}{l}\text { Easy to use; highly portable; } \\
\text { low cost; no sample } \\
\text { preparation needed }\end{array}$ & $\begin{array}{l}\text { Low separation } \\
\text { capability; sensors } \\
\text { drift overtime; } \\
\text { background VOC } \\
\text { interference }\end{array}$ & Yes \\
\hline Portable GC [34] & $\begin{array}{l}\text { Portable GC; Proposed } \\
\text { portable 2D GC; }\end{array}$ & Sorbent trap & sub-ppb & Moderate & $\begin{array}{l}\text { Portable; fully automated; } \\
\text { high separation capability; } \\
\text { high sensitivity; no } \\
\text { sample preparation } \\
\text { needed }\end{array}$ & $\begin{array}{l}\text { More complicated than } \\
\text { 1D portable GC }\end{array}$ & Yes \\
\hline
\end{tabular}

${ }^{\text {a }}$ Flame ionization detector/thermal conductivity detector

${ }^{\mathrm{b}}$ Field asymmetric ion mobility spectrometry

${ }^{\mathrm{c}}$ Gold nanoparticles

${ }^{\mathrm{d}}$ Carbon nanotubes

${ }^{\mathrm{e}}$ Conducting polymers

${ }^{\mathrm{f}}$ Parts-per-trillion, $10^{-12}$

${ }^{\mathrm{g}}$ Parts-per-billion, $10^{-9}$

${ }^{\mathrm{h}}$ Parts-per-million, $10^{-6}$

practice, breath from a subject is collected in a thermal desorption tube or sampling bag, and then transferred to GC-MS by thermal desorption device or by solid phase microextraction (SPME). Comprehensive 2-dimensional (2D) GC has improved the peak capacity over the traditional GC [37, 38]. VOC analytes are subject to two independent separation processes, first by their vapor pressures in the 1st-dimensional column and then by their polarities in the 2 nd-dimensional column. It has also been used for detection of diseases such as cancer, tuberculosis, and human volatome [39-41]. Due to the bulky size and the long turn-around times, GC-MS and comprehensive 2D GC are not suitable for POC applications and cannot be used to continuously monitor the subject to detect dynamic changes. SIFT-MS (selected-ion flow-tube mass spectrometry) and PTR-MS (proton transfer reaction tube mass spectrometry) has high sensitivity and can be used for real-time breath VOC monitoring [25-27]. However, the bulky size, heavy weight ( $>200 \mathrm{~kg}$ ), and high cost limit its wide acceptance. Ion mobility spectrometry (IMS) [29-31] can be operated under ambient pressure, thus avoid the use of a cumbersome vacuum pump. The portability and short analysis time (usually a few minutes) makes IMS suitable for POC application. Recently exploratory tests using FAIMS (Field Asymmetric Ion Mobility Spectrometry) technology in diagnosis of lung cancer, asthma, and inflammatory bowel disease have been reported [30, 31]. However, its limited VOC separation capability may affect the diagnostic accuracy. Electronic nose (e-nose) relies on varied vapor sensor arrays (such as colorimetric, gold nanoparticles, carbon nanotubes) and pattern recognition for breath analysis [21, 32, 33]. 
Although portable, fast, and easy to use, e-nose has poor chemical selectivity, device-to-device repeatability, and stability, as well as high susceptibility to background or interference VOCs $[21,22]$. Portable GC systems are also used in breath analysis [42]. However, current commercial portable GC systems are 1D devices and have limited separation capability (or peak capacity), which, again, may affect the diagnostic accuracy for given diseases. In addition, most of the 1D GC devices are not customized to operate in a fully automated mode, which hinders its clinical applications.

Recently, we have developed a fully automated portable GC device that can be operated simultaneously as 1D GC and comprehensive 2D GC with a sub-ppb sensitivity [43]. With the help of the 2-dimensional separation, the co-eluted peaks that are not separated from the 1st-dimensional column can further be separated on the 2nd-dimensional column, thus increasing device's separation capability. The aim of this study was to further adapt this portable GC for the use on a mechanical ventilator in ICUs and develop the related algorithms for rapid analysis of breath from patients undergoing mechanical ventilation, in order to understand the ability of our GC (and the algorithms) to detect the presence of ARDS compared to clinician adjudication.

Figure 1 shows the schematic of the GC device connected to a ventilator. In our work breath was collected and analyzed every $33 \mathrm{~min}$ via a small tube connected to the exhalation port of the ventilator. A total of 97 peaks were separated out from human breath. Through machine learning, principal component analysis (PCA), and linear discriminant analysis (LDA), 9 out of 97 peaks were selected as a VOC subset for the discrimination between ARDS and non-ARDS respiratory failure. Forty-eight (48) ARDS and non-ARDS patients with a total of 85 different breath chromatograms were evaluated. Among all 48 patients, we used 28 patients (43 sets of breath) as the training set and 20 patients (42 sets of breath) as the testing set. Using blinded physician adjudication of the patients' records based on the Berlin criteria as the gold standard, our breath analysis achieved an overall accuracy of $87.1 \%$ with $94.1 \%$ positive predictive value and $82.4 \%$ negative predictive value.

\section{Methods}

\section{Patient selection and diagnosis}

This study was approved by the University of Michigan Institutional Review Board (IRB) to consent adult patients 18 years or older undergoing mechanical ventilation for both hypoxic and non-hypoxic respiratory failure or need for mechanical ventilation for other life-support issues in various intensive care unit settings. Etiologies for the need for intubation and mechanical ventilation included ARDS, pneumonia, sepsis, pulmonary embolism, traumatic brain injury, cardiac arrest, severe chronic obstructive pulmonary disease exacerbations, and combinations of several of these reasons.

The final diagnosis of ARDS was adjudicated retrospectively by a multi-physician panel blinded to portable GC data. The adjudication was based on the Berlin criteria [9], which relies on a combination of medical history, chest radiography findings, and oxygenation parameters [9]. Details regarding this ARDS adjudication process have been previously reported [44].

An adjudication was performed for the day the patient was studied using the portable GC. If a patient was studied at more than one-time point, a separate adjudication was made on those days. The adjudication of ARDS was binary (present/ not present) and no attempt was made to score ARDS (if present) as mild, moderate, or severe. If patient subjects were successfully liberated from mechanical ventilations, no additional GC testing was performed.

In order to identify and populate the algorithm with breath signatures from individuals with no acute illness or injury requiring mechanical ventilation, we also recruited five laboratory members with no history of pulmonary conditions or acute illness as volunteer controls (denoted as patient \#1, 2, 3,
Fig. 1 Conceptual illustration of using a portable GC device to analyze breath from a patient on a mechanical ventilator
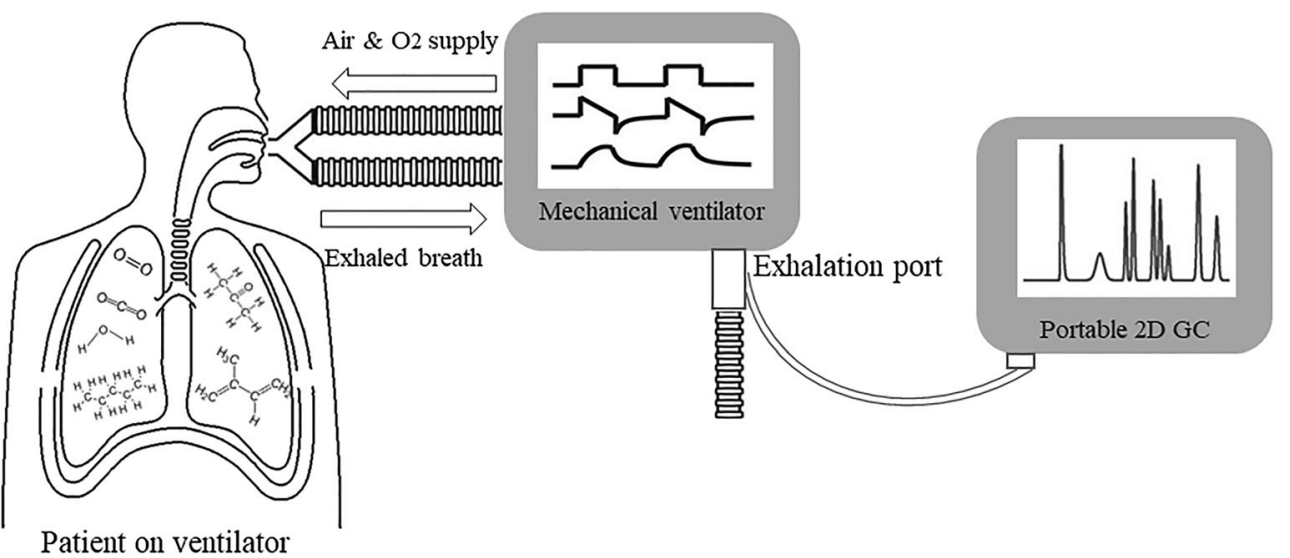

Patient on ventilator 
4, and 38 in Fig. 7 and Electronic Supplementary Material (ESM) Figs. S4 and S6). Their breath samples were collected in Tedlar bags through a moisture filter and then immediately withdrawn into the GC device for analysis. Patient \#1, 2, 3, and 4 were used in the training set whereas patient \#38 was used in the testing set.

A total of 21 ARDS patients and 27 non-ARDS control patients were recruited for 85 sets of breath chromatograms.

IRB statement This clinical research study (HUM00103401) was approved by the University of Michigan Medical School's Institutional Review Board (a component of the University of Michigan's Human Research Protection Program). Consent was required from patient subjects or their legally authorized representative prior to enrollment.

\section{Separation and detection of breath with the portable GC device}

As shown in Fig. 2, patient's breath was collected via a 2-m long polytetrafluoroethylene (PTFE) tubing $(0.64 \mathrm{~cm}$ i.d.) connected to the exhalation port of the ventilator. In order not to break the already-established respiratory circuits of the recruited patients, we adapted the direct sampling method (sampling from the ventilator exhalation port) rather than the end-tidal air collection method where a $\mathrm{CO}_{2}$ sensor is needed close to the tracheal tube to monitor the real-time $\mathrm{CO}_{2}$ concentration. As a result, the collected breath sample contains some dead space volume. The sampling rate was $70 \mathrm{~mL} / \mathrm{min}$ and the sampling time was $5 \mathrm{~min}$. The total assay time was $33 \mathrm{~min}$, which included $5 \mathrm{~min}$ of sample collection time, $5 \mathrm{~min}$ of desorption/transfer time, 13 min of separation time, and $10 \mathrm{~min}$ of cleaning time.

In Fig. 3, we give brief description of the portable $1 \times 2$ channel 2D GC and its operation. The details can be found in ESM Section S1. The 2D GC device consisted of three detachable modules: sampling module, 1st-dimensional separation module, and 2nd-dimensional separation module. The 1st-dimensional module further consisted of a home-made micro-thermal injector $(\mu \mathrm{TI})$, a 10-m long on-polar DB-1 ms column $(250 \mu \mathrm{m} \times 0.25 \mu \mathrm{m}$, Agilent J\&W Scientific), and a home-made micro-photoionization detector ( $\mu \mathrm{PID})$ [45]. The 2nd-dimensional module consisted of two identical channels, each of which had a 3-m long polar SUPELCOWAX® 10 column $(250 \mu \mathrm{m} \times 0.25 \mu \mathrm{m}$, Sigma Aldrich). Note that while polar columns have been used in the 2nd-dimensional column in 2D GC analysis of breath [39, 40], mid-polar columns can also be used [41]. The $1 \times 2$-channel $2 \mathrm{D} \mathrm{GC}$ can be operated as a $1 \mathrm{D}$ GC alone when the 2 nd-dimensional module is either disabled or detached or as comprehensive 2D GC. To increase the separation capability, in this work, we chose to operate our portable GC in a comprehensive 2D GC mode, which required additional but negligible $20 \mathrm{~s}$ compared to $1 \mathrm{D}$ GC operation alone. In the comprehensive 2D GC mode, eluted analytes from the ${ }^{1} \mathrm{D}$ column were sliced by the micro-Deans switch, loaded onto the one of the $\mu$ TIs ( $\mu$ TI 2A or $\mu$ TI 2B in Fig. 3), and then injected into the corresponding ${ }^{2} \mathrm{D}$ column $\left({ }^{2} \mathrm{D}\right.$ column $2 \mathrm{~A}$ or ${ }^{2} \mathrm{D}$ column $2 \mathrm{~B}$ in Fig. 3). The modulation time was $10 \mathrm{~s}$ so that the maximally allowed separation time on each ${ }^{2} \mathrm{D}$ column was $20 \mathrm{~s}$ [43]. The ${ }^{1} \mathrm{D}$ column was programmed to start from $25{ }^{\circ} \mathrm{C}$ for $2 \mathrm{~min}$ and was then heated to $80{ }^{\circ} \mathrm{C}$ with a ramping rate of $10{ }^{\circ} \mathrm{C} / \mathrm{min}$. Then the
Fig. 2 Detailed description of the experimental setup. The portable $\mathrm{GC}$ was connected to the output of a ventilator via a $2 \mathrm{~m}$ long polytetrafluoroethylene (PTFE) tubing $(0.64 \mathrm{~cm}$ i.d.). The portable GC weighed less than $5 \mathrm{~kg}$. The patient breath was drawn into and captured by the thermal desorption tube in the GC device at a flow rate of $70 \mathrm{~mL} / \mathrm{min}$ for $5 \mathrm{~min}$. The total assay time was $33 \mathrm{~min}$, which included 5 min of sample collection time, $5 \mathrm{~min}$ of desorption/transfer time, 13 min of separation time, and 10 min of cleaning time. For details of GC operation, see the ESM

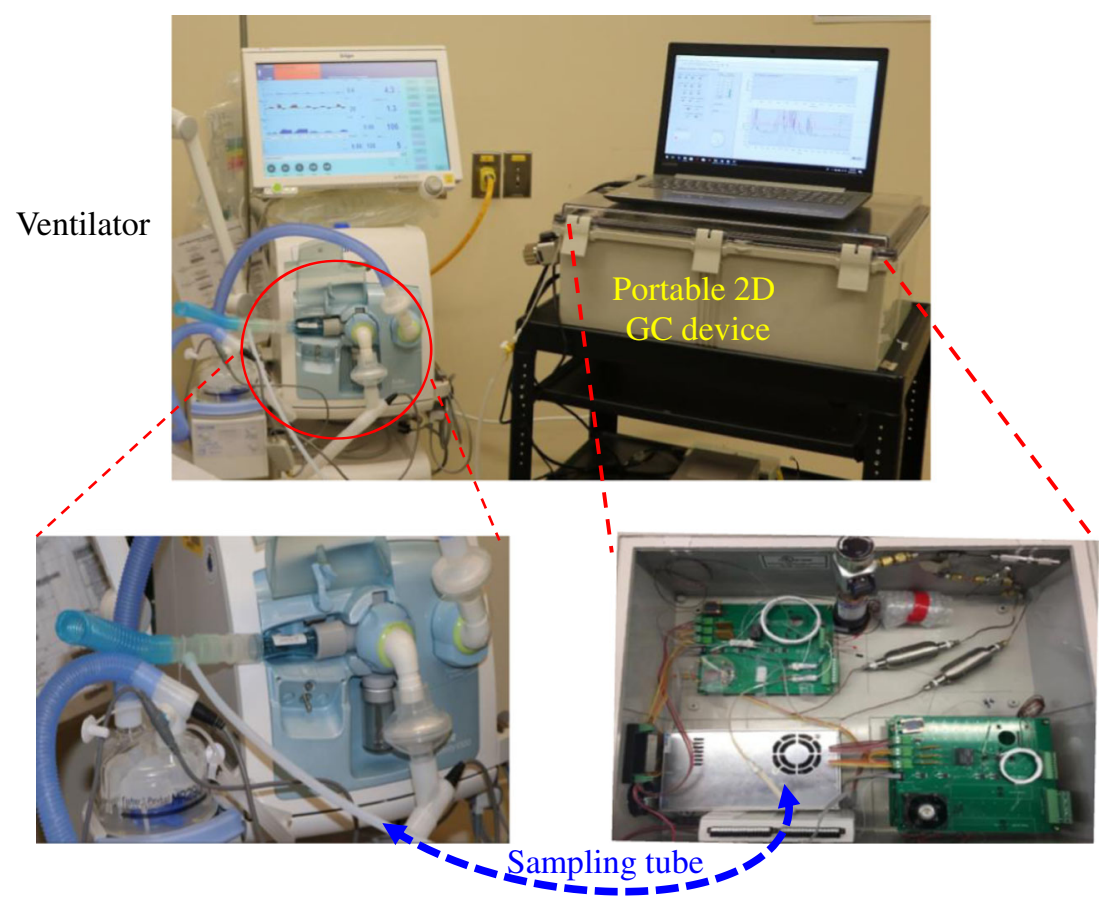




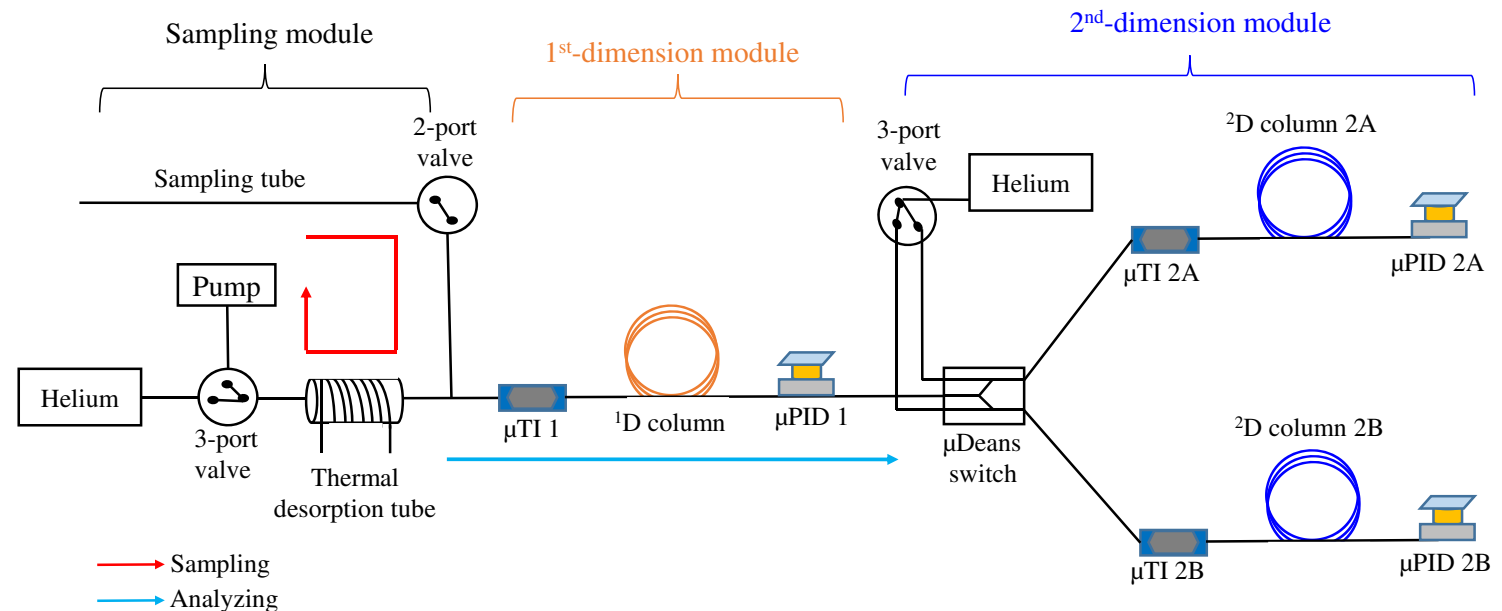

Fig. 3 Layout of the portable $1 \times 2$-channel 2D GC device. It consisted of three detachable modules: sampling module, 1st-dimensional separation module, and 2nd-dimensional separation module. The 1st-dimensional module consisted of a micro-thermal injector ( $\mu \mathrm{TI})$, a non-polar column, and a micro-photoionization detector ( $\mu$ PID). The 2nddimensional module had two identical channels, each of which consisted of a $\mu \mathrm{TI}$, a polar column, and a $\mu$ PID. During operation, breath was collected via the sampling tube and captured by the thermal desorption tube. Then the analytes were transferred to $\mu$ TI 1 and injected into the ${ }^{1} \mathrm{D}$ column. The elution from the ${ }^{1} \mathrm{D}$ column was detected by

temperature was raised to $120{ }^{\circ} \mathrm{C}$ with a ramping rate of $20{ }^{\circ} \mathrm{C} / \mathrm{min}$ and finally kept at $120{ }^{\circ} \mathrm{C}$ for $4 \mathrm{~min}$. Both ${ }^{2} \mathrm{D}$ columns were kept at $75^{\circ} \mathrm{C}$. In our $1 \times 2$-channel $2 \mathrm{D} \mathrm{GC}$, we used 3 flow-through $\mu$ PIDs, one at the end of the ${ }^{1} \mathrm{D}$ column ( $\mu$ PID 1 in Fig. 3) and two at the end of the ${ }^{2} \mathrm{D}$ columns ( $\mu$ PID 2A and $\mu$ PID $2 \mathrm{~B}$ in Fig. 3). The use of a detector at the end of ${ }^{1} \mathrm{D}$ column allows us to monitor the elution of the analytes from the ${ }^{1} \mathrm{D}$ column to produce the 1D chromatogram (if the GC device is operated as $1 \mathrm{D}$ GC alone) or to avoid potential under-sampling that may be caused by the $10 \mathrm{~s}$ modulation time (if the GC device is operated as comprehensive 2D GC) (see the detailed discussion in Ref. [43]).

Details of 2D GC chromatogram construction based on the signal obtained by the 3 detectors and the algorithm for the subsequent analysis are described in ESM Sections S2 and S3, respectively.

\section{Results}

\section{Chromatograms for ARDS and non-ARDS patients}

Figure 4 shows the representative 1D and 2D chromatograms for an ARDS and a non-ARDS control. It can be seen from Fig. 4 and ESM Fig. S1 that 2D GC provides additional separation capability as compared to the 1D GC (3-10 times higher in terms of peak capacity according to the analysis in ESM Section S4). $\mu \mathrm{PID} 1$. If 2nd-dimensional separation was needed, the 2nd-dimensional module was attached to the outlet of the ${ }^{1} \mathrm{D}$ column and the elution from the ${ }^{1} \mathrm{D}$ column was sliced and sent alternately to one of the two ${ }^{1} \mathrm{D}$ columns via a micro-Deans switch. The portable GC can operate as $1 \mathrm{D}$ $\mathrm{GC}$ alone when the 2nd-dimensional module is disabled or detached, or as comprehensive 2D GC. Comprehensive 2D GC operation required additional $20 \mathrm{~s}$ compared to $1 \mathrm{D}$ GC operation alone, which was negligible considering the overall assay time of $\sim 30 \mathrm{~min}$. For details of GC operation and 2D GC chromatogram construction, see the ESM

Figure 5 shows that a total 97 peaks were found collectively in the $852 \mathrm{D}$ chromatograms from the patients under study, among which nearly $70 \%$ of the peaks are co-eluted or partially co-eluted in the ${ }^{1} \mathrm{D}$ column. Note that not all 97 peaks appear in a 2D chromatogram for a particular patient, as some peaks are not present in that patient's breath or below the detection limit of our $\mu$ PIDs. Although our portable 2D GC does not generate as many peaks as the high-end benchtop 2D GC in tandem with MS [41], it can still distinguish ARDS and non-ARDS as shown later. Also, among all the recruited and adjudicated patients, we were able to monitor 9 ARDS patients and 9 non-ARDS patients for multiple days.

Figure 6 shows, as an example, the 2D chromatograms for an ARDS patient tested over 3 days, from which we can see clearly that breath VOC peaks changes qualitatively and quantitatively (for example, peak \#2 and \#44). ARDS trajectory tracking is given in "Time series measurement of ARDS patients."

\section{Patient classification based on 2D chromatograms}

Among all 97 peaks, not all of them appear to be relevant to ARDS. For example, some of the peaks may be from indoor air background, normal metabolic activities, or other conditions that a patient may have. Those irrelevant peaks interfere in the correct classification of ARDS and non-ARDS groups. It is therefore critical to determine which subset of the peaks is most responsible for the differences observed between ARDS and non-ARDS groups. To select the optimal subset of peaks, 

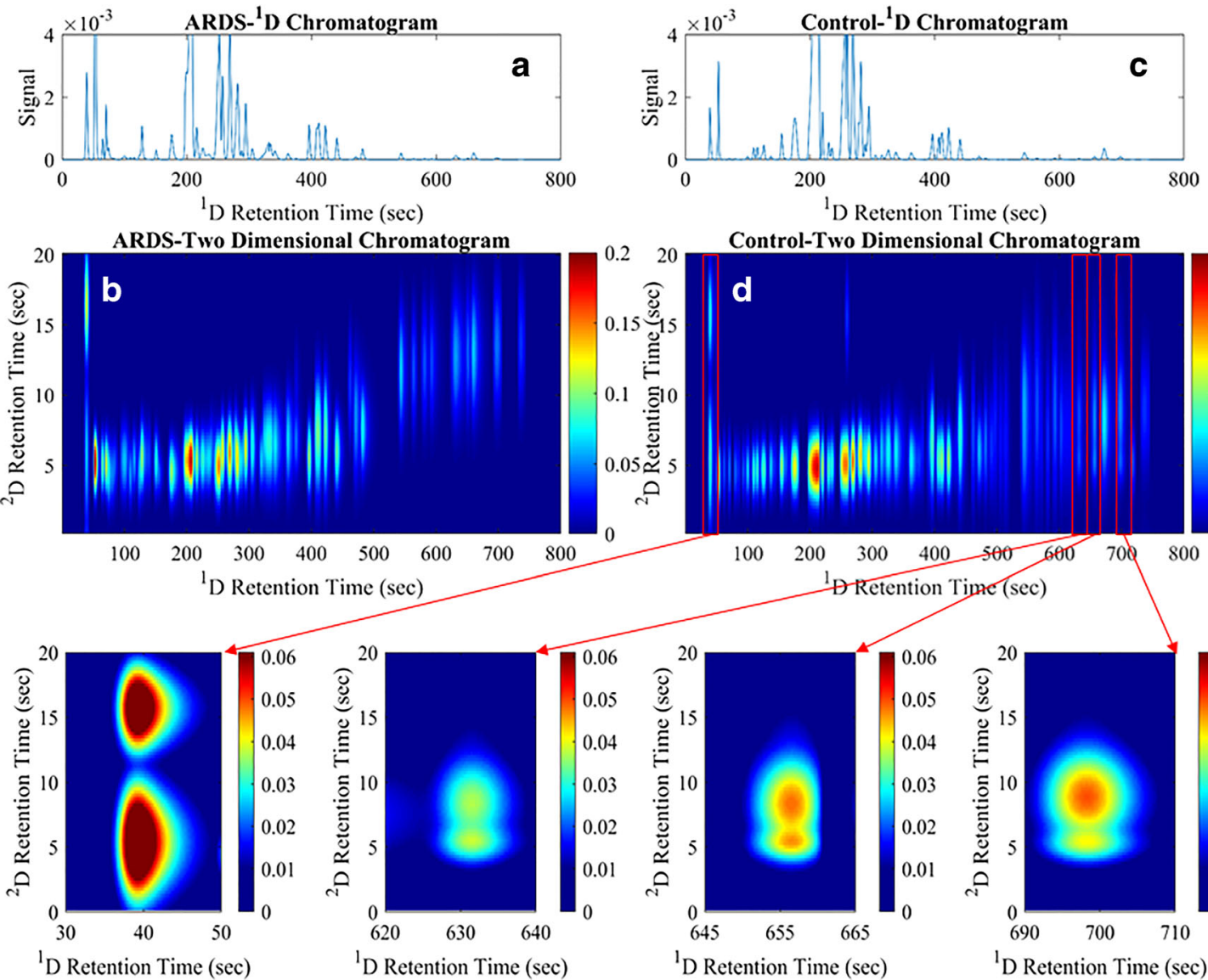

Fig. 4. a, b Representative 1D chromatogram and 2D chromatogram for an ARDS patient, respectively. c, $\mathbf{d}$ Representative 1D chromatogram and 2D chromatogram for a non-ARDS (control) patient, respectively. In the

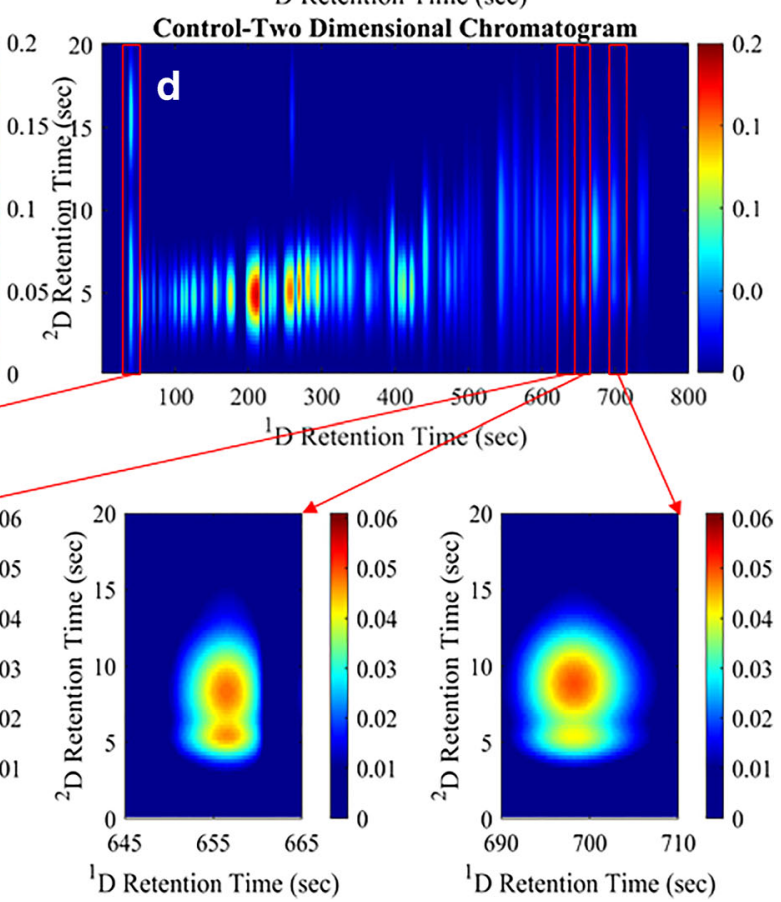

zoomed-in 2D chromatogram for the control patient, four co-eluted ${ }^{1} \mathrm{D}$ peaks are separated into eight peaks in the $2 \mathrm{D}$ chromatogram. Other zoomed-in portions of $\mathbf{b}$ and $\mathbf{d}$ can be found in ESM Fig. S1

28 patients (11 ARDS, 17 control, and a total of 43 tests) were used as the training set, whereas the remaining 20 patients (10
ARDS, 10 controls, and a total of 42 tests) were used as the testing set.

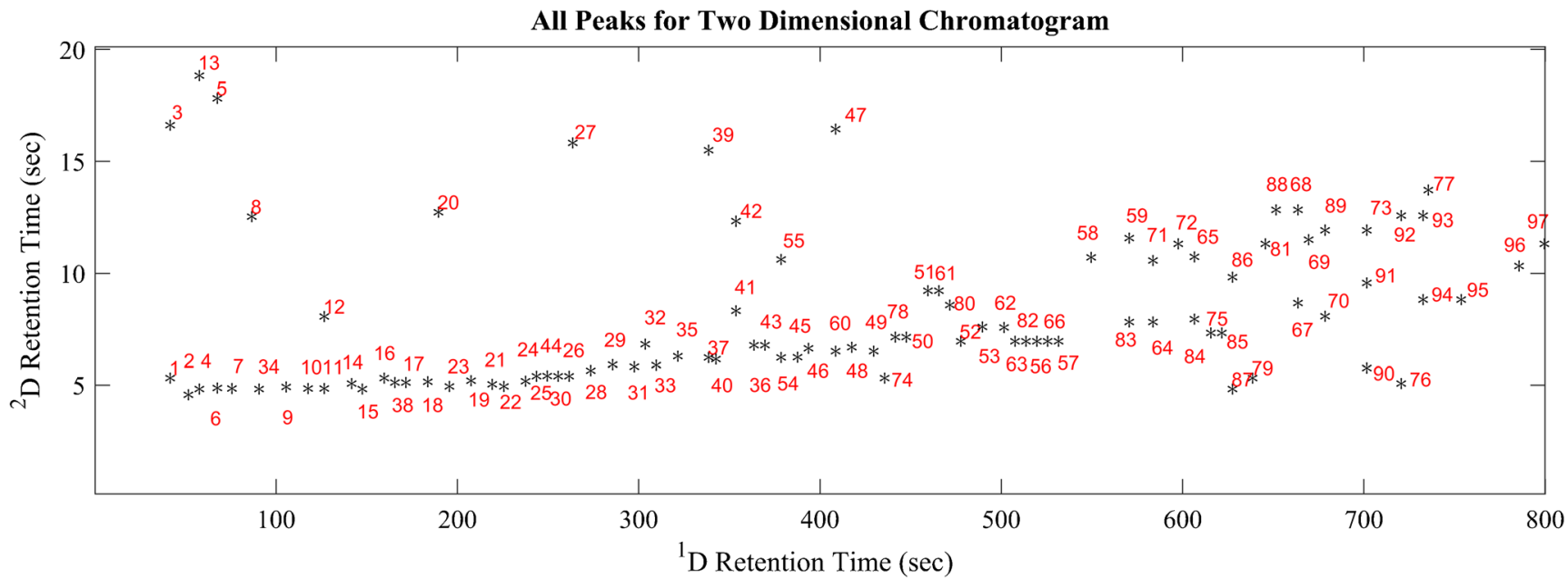

Fig. 5 All 97 peaks found collectively in 85 breath samples from 48 patients plotted in a 2D chromatogram, among which 18 pairs (36 peaks) are co-eluted and approximately another 30 peaks are partially co-eluted (with doublets or triplets and separation of adjacent peaks is less than $2 \sigma$ ) from the ${ }^{1} \mathrm{D}$ column. Each dot represents the center of a peak in the contour plot (see, for example, Fig. 4, for a peak contour plot). Note that not all 97 peaks appear in a $2 \mathrm{D}$ chromatogram for a particular patient 
Fig. 6 Evolution of the 2D chromatogram of an ARDS patient (patient \#11) during 3 days of monitoring
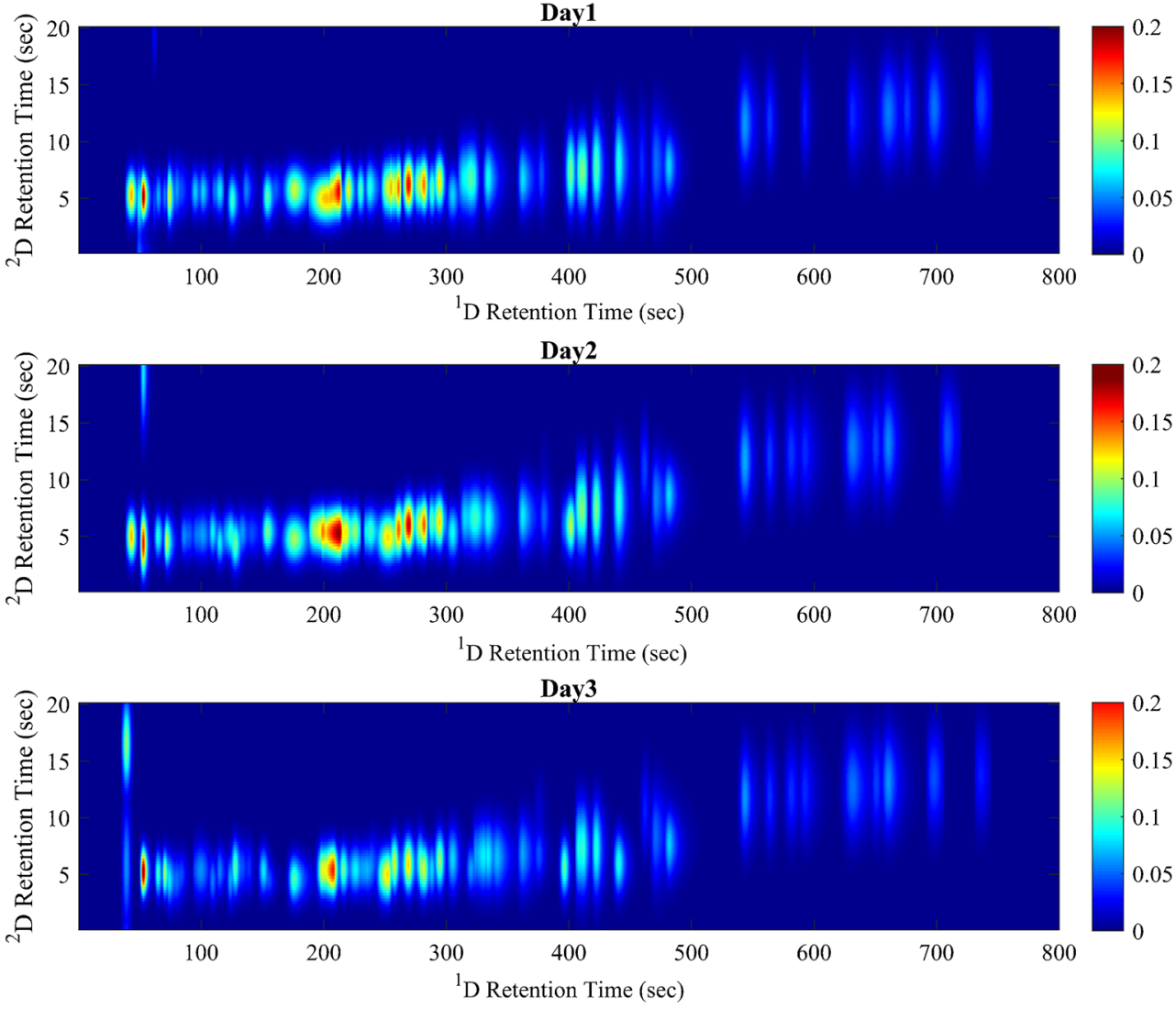

An algorithm based on machine learning, principal compowas developed to first select the optimal subset of peaks using the training set. Then with the PCA coefficients acquired from
Fig. 7 PCA plot of all recruited patients. $X$-axis (PC 1 ) is the 1 st principal component and $Y$-axis (PC 2) is the 2nd principal component. The red and black symbols denote respectively the ARDS and non-ARDS patients adjudicated by physicians using the Berlin criteria. The patient numbers are given by the symbol. For example, "11.1" and "11.3" denote patient \#11, day 1 and day 3 results, respectively. The bottom/top zone below/above the boundary line represents respectively the ARDS/nonARDS region using the breath analysis method. The corresponding Q-residuals for this PCA model are shown in ESM Fig. S5

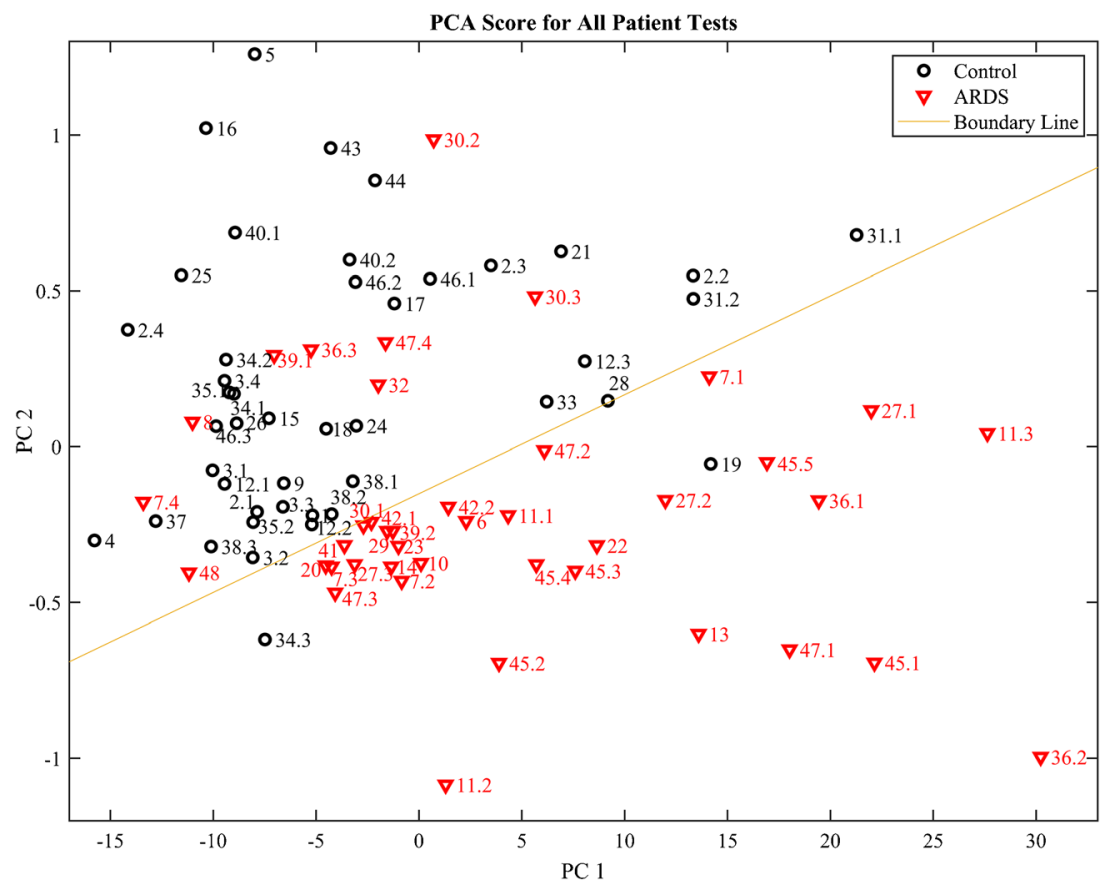


Table 3 Statistics of breath analysis for ARDS

\begin{tabular}{lccc}
\hline & ARDS & $\begin{array}{l}\text { Non- } \\
\text { ARDS }\end{array}$ & Total \\
\hline Positive (our results) & 32 & 2 & 34 \\
Negative (our results) & 9 & 42 & 51 \\
Column total & 41 & 44 & 85 \\
Specificity & $95.5 \%$ & & \\
Sensitivity & $78.0 \%$ & & \\
Positive predictive value & $94.1 \%$ & & \\
Negative predictive value & $82.4 \%$ & & \\
Total accuracy & $87.1 \%$ & & \\
\hline
\end{tabular}

the training set, the PCA scores for the testing set of patients can be calculated. The details of the algorithm are given in ESM Section S3. With this algorithm, we selected 9-peak subset as the final optimal peak subset, which yields the best classification accuracy (93.0\%) and the maximum boundary distance. The final PCA scores for all recruited patients are shown in Fig. 7. The final PCA model achieved an overall accuracy of $87.1 \%$ with $94.1 \%$ positive predictive value and $82.4 \%$ negative predictive value. The corresponding specificity, sensitivity, positive predictive value (PPV), and negative predictive value (NPV) are presented in Table 3. The corresponding Q-residuals for all recruited patients are shown in ESM Fig. S5. Separate PCA scores and the corresponding statistics (specificity, sensitivity, PPV, and NPV) for the training and testing sets are presented in ESM Fig. S6 and Table S2, respectively. Receiver operating characteristic curves for the training set, testing set, and all patients are presented in ESM Section S3.6 and Fig. S7. The 4-fold cross-validation was performed, where the original datasets were randomly divided into 4 subsets of equal size and 4 cross-validation models were generated using one subset as testing set and the rest as training set. The 4 models yielded a classification accuracy of $85.3 \% \pm 0.7 \%$, which indicates the robustness of the model. The statistics (specificity, sensitivity, PPV, and NPV) of the 4 models are presented in ESM Table S3.

\section{Time series measurement of ARDS patients}

One of important advantages of breath analysis is the potential to non-invasively monitor the development of ARDS, the severity of ARDS (if present), and the resolution of ARDS. This would allow the technology to map the trajectory of the disease and potentially guide therapy and decision making. Among the 9 ARDS patients and 9 non-ARDS patients whom we monitored on multiple days, some ARDS patients were noted to clinically progress to a non-ARDS status and vice versa, as determined by both 2D GC and clinical adjudication.
Fig. 8 The trajectory on PCA plot for patient \#11,\#27, \#36, and \#47. $\# 11$ and \#27 are the upgrade case (initially listed as potential ARDS on the first day) and \#36 and \#47 are recovery cases (extubated and discharged from ICU $24-48 \mathrm{~h}$ after the last test). The bottom/top zone below/above the boundary line represents respectively the ARDS/non-ARDS region using the breath analysis method

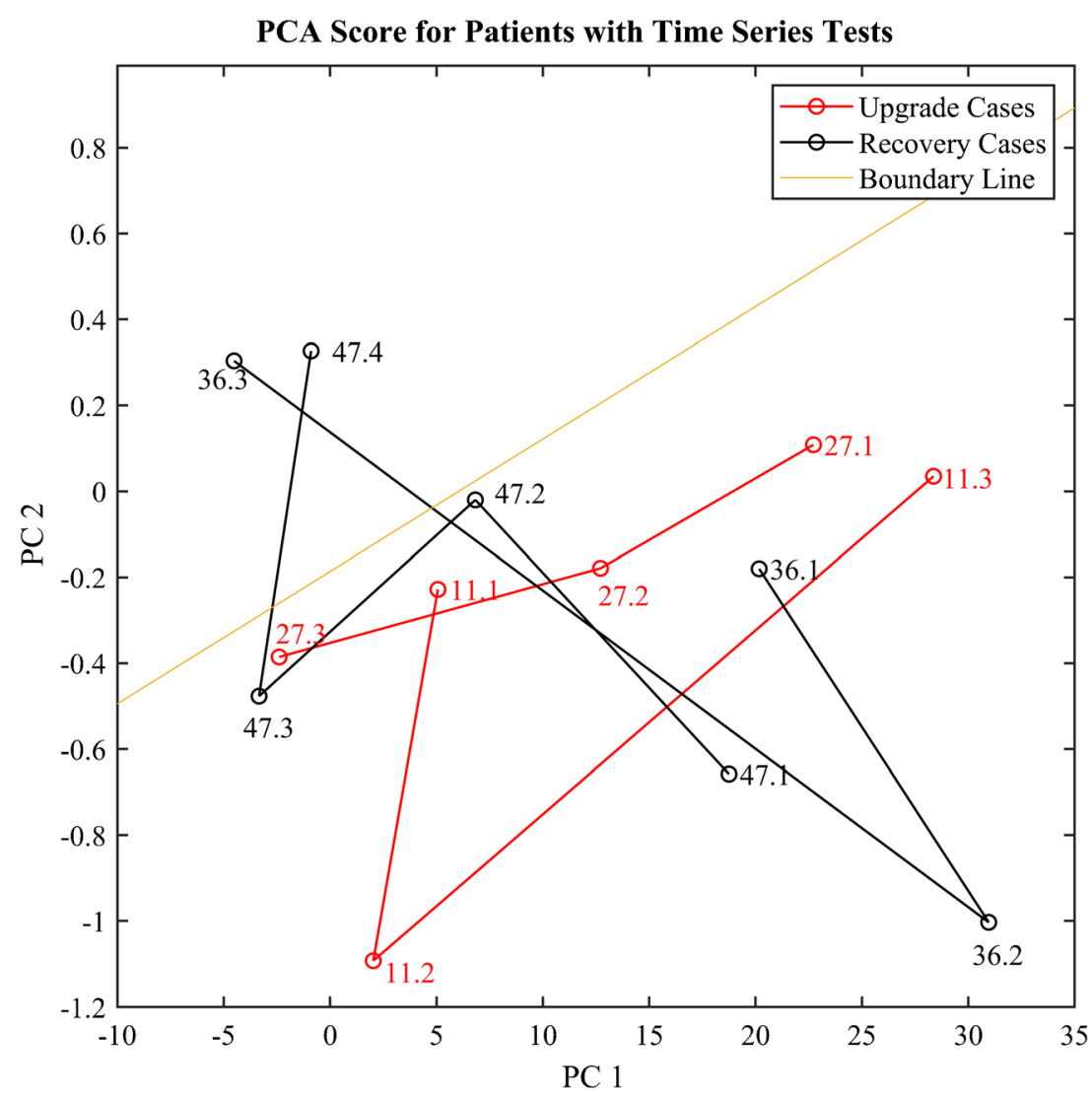


Our results demonstrated that breath analysis may be able to predict the ARDS trajectory $12-48 \mathrm{~h}$ in advance. Below we show some examples, whose trajectories on the PCA plot are shown in Fig. 8.

\section{Upgrade cases}

Patient \#11 (see also Fig. 6 for the corresponding time series chromatograms) was a potential and undetermined ARDS patient (meaning that the clinician suspected that the patient might develop ARDS, but was not certain at the time of diagnosis. The patient was placed on close monitoring) on the $1 \mathrm{st}$ test day and then upgraded to ARDS on the next day. The breath test suggested ARDS from the 1st test day to the $3 \mathrm{rd}$ test day (\#11.1,\#11.2, and \#11.3).

Patient \#27 was a potential and undetermined ARDS patient on the 1st test day and then upgraded to ARDS on the next day. The breath test suggested ARDS from the 1st test day to the 3rd test day (\#27.1, \#27.2, and \#27.3).

\section{Recovery cases}

Patient \#36 was sampled for 3 days. On the 3rd day, the patient was still listed as ARDS patients based on the Berlin criteria and then got extubated (liberated from mechanical ventilation) and discharged from ICU on the 5th day. The breath tests for the first 2 days suggested ARDS (\#36.1 and \#36.2). The breath test for the 3 rd day demonstrated a non-ARDS pattern (\#36.3).

Patient \#47 was sampled for 4 days and was liberated form mechanical ventilation and discharged on the 6th day. Based on the Berlin criteria, this patient had ARDS for all first 4 days. The breath tests for the first 3 days breath tests show an ARDS pattern (\#47.1, \# 47.2, and \#47.3) and the 4th day breath test shows as non-ARDS pattern (\#47.4).

If the breath test results do not match the clinical adjudication, we consider the test as "misclassification" when calculating the overall classification accuracy, even for the cases like \#36.3 and \#47.4 that suggest that the breath analysis was able to predict the trajectory of ARDS (i.e., earlier diagnosis).

With further evidence on the following days, the two potential and undetermined ARDS cases mentioned above (\#11.1 and \#27.1) were finally determined as ARDS based on the Berlin criteria.

The trajectories of the entire 18 patients and their medical histories can be found in ESM Fig. S8 and Section S5.

\section{Discussion}

To our knowledge, the portable 2D GC device described here is the first of its kind that can be used in POC to continuously monitor patient breath. Using this portable GC device, along with algorithms developed in this work, we are able to distinguish ARDS and non-ARDS with high accuracy, compared to clinical expert adjudication. As a dynamic syndrome with multiple etiologies, the real-time diagnosis of ARDS is challenging. There are currently no technologies allowing its realtime diagnosis or tracking. The only widely available tool in use in assisting in ARDS diagnostics is LIPS. However, LIPS was designed as a screening tool that incorporates a series of risk factors and risk modifiers to predict whether ARDS will occur at a future point. While a small subset of data using the 2D GC technology indicates the potential to predict onset or resolution, much more testing will be needed. An interesting possibility would be to utilize LIPS in conjunction with the technology to improve screening $[20,22]$.

It should be noted that based on the results obtained through the current work, the 9-peak subset (peak \#2, 34, $38,44,62,66,72,79$, and 81 in Fig. 5) that was selected for ARDS detection can be almost separated using the ${ }^{1} \mathrm{D}$ column in our current 2D GC device (except peak \#34 that is nearly co-eluted with peak \#8. See ESM Fig. S3). Therefore, it seems that if we only need to distinguish between ARDS and nonARDS, then our portable GC can simply be operated in a 1D GC mode alone. However, we believe that 2D GC operation is still a preferred choice, since the potential co-elution of peak \#34 and \#8 may affect the ARDS detection. More significantly, 2D GC operation is critical to sub-typing ARDS and analyzing complications. For example, the peaks (\#3, 5, 13, and 27 ) in Fig. 5 do not belong to the ARDS-relevant 9-peak subset, but they have different concentrations between the ARDS patient and the healthy control (see Fig. 4b, d and ESM Fig. S1), suggesting that the ARDS patient in Fig. 4b might have other health conditions besides ARDS. In addition, the peaks (\#3, 5, and 13) in Fig. 6, which are not part of the 9-peak subset, indicate other health conditions (besides ARDS) of the same patient might change during medical treatment of ARDS. For future applications (in detection of ARDS and ARDS with complications, and in detection of other diseases such as asthma and pneumonia), it is still preferred to continue to use 2D GC to separate as many peaks as possible, which makes the device much more flexible for various diseases and medical conditions rather than being dedicated to monitoring of ARDS alone.

This study has a number of important limitations. First and foremost is that the histopathologic examination of lung tissue for changes consistent with diffuse alveolar damage (DAD) was not used to make the diagnosis of ARDS. Even in those patients dying of respiratory failure, autopsies were not obtained. While DAD on histopathology is the pathologic gold standard, obtaining serial lung biopsies for diagnosis is not feasible or a clinical standard of care. Although the clinical consensus for the diagnosis of ARDS is the Berlin criteria, Kao and others have demonstrated that of patients clinically 
diagnosed as having ARDS using the Berlin criteria, less than $60 \%$ have DAD on lung histopathology when lung biopsies can be obtained [11]. In the absence of tissue biopsy, we employed the best available instrument (multi-physician adjudication) for identifying ARDS [44].

While this is a limitation to our study, it is also a limitation to any clinical research or clinical trial in the field of ARDS further underscoring the need for new diagnostics. Despite this, we noted the VOC patterns that where clearly distinguishable from patterns seen in subjects who were mechanically ventilated for non-hypoxic respiratory failure such as sepsis (without ARDS) and cardiac arrest, as well as those intubated for hypoxic respiratory failure in whom after intubation and mechanical ventilation, the patient's $\mathrm{PaO}_{2} / \mathrm{FiO}_{2}$ was clearly not indicative of ARDS (COPD exacerbation, pulmonary embolism, and unilateral pneumonia). In cases of divergence in clinical scoring and breath analysis result, differences could be due to missdiagnosis of ARDS by clinical scoring, the ability of breath analysis to detect earlier onset or resolution of ARDS than clinical adjudication, mixed lung and systemic pathologies existing in the same patients, and others.

Finally, we note that in the current study, only 48 patients (and 85 sets of breath samples) were used for the proof of the concept. A much larger groups of patients need to be recruited to further validate our method. Additionally, the selected 9 peaks as well as other peaks that may be relevant to lung and systemic pathologies should all be identified in order to understand the underlying physiological conditions of the patients and how those VOCs are involved in the ARDS processes.

\section{Conclusions}

We have developed an automated portable 2D GC device and a corresponding algorithm for breath analysis that begins to enable distinguishing the condition of ARDS from nonARDS. Particularly, the $94.1 \%$ positive predicative value suggests that our breath analysis method can accurately diagnose ARDS, which is critical to its treatment. In several subjects studied, the technology was found to indicate the presence of ARDS prior to the development of traditional indicators used for ARDS diagnosis opening up the potential for earlier interventions. The non-invasive nature of breath analysis may also allow for continuous monitoring of ARDS trajectory as evidenced by several subjects who demonstrated changing breathomic patterns from ARDS to non-ARDS status prior to changes in traditional indicators.

The potential to leverage exhaled breath for the identification of breathomic patterns used for early diagnosis, disease trajectory tracking, and outcome prediction monitoring of ARDS would have significant impact on changing practice and improving patient outcomes. The device is envisioned to be used for ARDS patients in emergency departments, operating rooms, and intensive care units. Additionally, our device holds the potential to dramatically improve the molecular characterization of ARDS and its competing diagnoses. This clinical ambiguity of the diagnosis of ARDS compared with histopathology impairs the field's ability to develop and study targeted, disease-specific therapies. Exhaled breath VOC analysis could significantly enhance our molecular phenotyping of patients with hypoxic respiratory failure, crystallizing our diagnoses, and dramatically improving our ability to tailor treatments to and enrich clinical trials with patients with true ARDS pathophysiology.

The preprint of this work that contained the preliminary results was published at bioRxiv [46], which can be accessed at https://www.biorxiv.org/content/10.1101/560888v1.

Acknowledgments They thank the support from Flux HPC Cluster provided by the University of Michigan Office of Research and Advanced Research Computing-Technology Services (ARC-TS), and Analytical Chemistry Lab at the University of Michigan Biological Station at Pellston, Michigan, for using their mass spectrometry facility.

Funding information The authors received financial support from National Institutes of Health (1-R21-HL-139156-01), NIH Center for Accelerated Innovations at Cleveland Clinic (Program Prime Award Number: 1UH54HL119810-05; Project Number: NCAI-17-7-APPUMICH-Fan), the Michigan Translation and Commercialization (MTRAC) for Life Sciences Hub, and the Michigan Center for Integrative Research in Critical Care.

\section{Compliance with ethical standards}

Conflict of interest The authors declare that they have no conflict of interest.

Research involving human participants and/or animals Research involves human participants. The clinical research study (HUM00103401) was approved by the University of Michigan's Human Research Protection Program.

Informed consent Consent was obtained from patient subjects or their legally authorized representatives by means of a voluntarily completed consent form prior to enrollment.

\section{References}

1. Rubenfeld GD, Caldwell E, Peabody E, Weaver J, Martin DP, Neff $\mathrm{M}$, et al. Incidence and outcomes of acute lung injury. N Engl $\mathrm{J}$ Med. 2005;353(16):1685-93.

2. Zambon M, Vincent JL. Mortality rates for patients with acute lung injury/ARDS have decreased over time. Chest. 2008;133(5):11201127.

3. Crader KM, Repine JJD, Repine JE. Breath biomarkers and the acute respiratory distress syndrome. J Pulmon Respir Med. 2012;2(1):111. 
4. Herridge MS, Tansey CM, Matte A, Tomlinson G, Diaz-Granados $\mathrm{N}$, Cooper A, et al. Functional disability 5 years after acute respiratory distress syndrome. N Engl J Med. 2011;364(14):1293-304.

5. Mikkelsen ME, Christie JD, Lanken PN, Biester RC, Thompson BT, Bellamy SL, et al. The adult respiratory distress syndrome cognitive outcomes study: long-term neuropsychological function in survivors of acute lung injury. Am J Respir Crit Care Med. 2012;185(12):1307-15.

6. Dasta JF, McLaughlin TP, Mody SH, Piech CT. Daily cost of an intensive care unit day: the contribution of mechanical ventilation. Crt Care Med. 2005;33:1266-71.

7. Hudson LD, Milberg JA, Anardi D, Maunder RJ. Clinical risks for development of the acute respiratory distress syndrome. Am J Respir Crit Care Med. 1995;151(2 Pt 1):293-301.

8. Gajic O, Dabbagh O, Park PK, Adesanya A, Chang SY, Hou P, et al. Early identification of patients at risk of acute lung injury. Am J Respir Crit Care Med. 2011;183(4):462-70.

9. Ranieri VM, Rubenfeld GD, Thompson BT, Ferguson ND, Caldwell E, Fan E, et al. Acute respiratory distress syndrome: the Berlin definition. JAMA. 2012;307(23):2526-33.

10. Thille AW, Esteban A, Fernandez-Segoviano P, Rodriguez JM, Aramburu JA, Penuelas O, et al. Comparison of the Berlin definition for acute respiratory distress syndrome with autopsy. Am J Respir Crit Care Med. 2013;187(7):761-7

11. Kao K-C, Hu H-C, Chang C-H, Hung C-Y, Chiu L-C, Li S-H, et al. Diffuse alveolar damage associated mortality in selected acute respiratory distress syndrome patients with open lung biopsy. Crit Care. 2015;19:228.

12. Carpenter CT, Price PV, Christman BW. Exhaled breath condensate isoprostanes are elevated in patients with acute lung injury or ARDS. Chest. 1998;114(6):1653-9.

13. Rahimpour E, Khoubnasabjafari M, Jouyban-Gharamaleki V, Jouyban A. Non-volatile compounds in exhaled breath condensate: review of methodological aspects. Anal Bioanal Chem. 2018;410(25):6411-40.

14. McNeil JB, Shaver CM, Kerchberger VE, Russell DW, Grove BS, Warren MA, et al. Novel method for noninvasive sampling of the distal airspace in acute respiratory distress syndrome. Am J Respir Crit Care Med. 2018;197(8):1027-35.

15. Alkhouri N, Singh T, Alsabbagh E, Guirguis J, Chami T, Hanouneh I, et al. Isoprene in the exhaled breath is a novel biomarker for advanced fibrosis in patients with chronic liver disease: a pilot study. Clin Transl Gastroenterol. 2015;6(9):1-7.

16. Miekisch W, Schubert JK, Noeldge-Schomburg GFE. Diagnostic potential of breath analysis-focus on volatile organic compounds. Clin Chim Acta. 2004;347:25-39.

17. Gordon SM, Szidon JP, Krotoszynski BK, Gibbons RD, O'Neill HJ. Volatile organic compounds in exhaled air from patients with lung cancer. Clin Chem. 1985;31(8):1278-82.

18. O'Neill HJ, Gordon SM, O'Neill MH, Gibbons RD, Szidon JP. A computerized classification technique for screening for the presence of breath biomarkers in lung cancer. Clin Chem. 1988;34(8):16138 .

19. Ware LB, Koyama T, Zhao Z, Janz DR, Wickersham N, Bernard $\mathrm{GR}$, et al. Biomarkers of lung epithelial injury and inflammation distinguish severe sepsis patients with acute respiratory distress syndrome. Crit Care. 2013;17(5):R253.

20. Bos LDJ, Weda H, Wang YY, Knobel HH, Nijsen TME, Vink TJ, et al. Exhaled breath metabolomics as a noninvasive diagnostic tool for acute respiratory distress syndrome. Eur Respir J. 2014;44(1): 188-97.

21. Bos LD, Schultz MJ, Sterk PJ. Exhaled breath profiling for diagnosing acute respiratory distress syndrome. BMC Pulm Med. 2014;14:72.

22. Bos LDJ. Diagnosis of acute respiratory distress syndrome by exhaled breath analysis. Ann Transl Med. 2018;6(2):33.
23. Phillips M, Cataneo RN, Saunders C, Hope P, Schmitt P, Wai J. Volatile biomarkers in the breath of women with breast cancer. J Breath Res. 2010;4(2):026003-11.

24. Phillips M, Basa-Dalay V, Bothamley G, Cataneo RN, Lam PK, Natividad MPR, et al. Breath biomarkers of active pulmonary tuberculosis. Tuberculosis. 2010;90(2):145-51.

25. Storer M, Curry K, Squire M, Kingham S, Epton M. Breath testing and personal exposure-SIFT-MS detection of breath acetonitrile for exposure monitoring. J Breath Res. 2015;9(3): 036006.

26. Smith D, Spanel P. SIFT-MS and FA-MS methods for ambient gas phase analysis: developments and applications in the UK. Analyst. 2015;140(8):2573-91.

27. Spanel P, Smith D. Progress in SIFT-MS: breath analysis and other applications. Mass Spectrom Rev. 2011;30(2): 236-67.

28. Moser B, Bodrogi F, Eibl G, Lechner M, Rieder J, Lirk P. Mass spectrometric profile of exhaled breath - field study by PTR-MS. Respir Physiol Neurobiol. 2005;145(2):295-300.

29. Cumeras R, Figueras E, Davis CE, Baumbach JI, Gràciaa I. Review on ion mobility spectrometry. Part 1: current instrumentation. Analyst. 2015;140:1376-90.

30. Covington JA, van der Schee MP, Edge AS, Boyle B, Savage RS, Arasaradnam RP. The application of FAIMS gas analysis in medical diagnostics. Analyst. 2015;140(20):6775-81.

31. https://www.owlstonemedical.com/. Accessed 3 July 2019.

32. Nakhleh MK, Amal H, Jeries R, Broza YY, Aboud M, Gharra A, et al. Diagnosis and classification of 17 diseases from 1404 subjects via pattern analysis of exhaled molecules. ACS Nano. 2017;11(1): 112-25.

33. Queralto N, Berliner AN, Goldsmith B, Martino R, Rhodes P, Lim SH. Detecting cancer by breath volatile organic compound analysis: a review of array-based sensors. J Breath Res. 2014;8(2):027112.

34. Cai X, Chen L, Kang T, Tang Y, Lim T, Xu M, et al. A prediction model with a combination of variables for diagnosis of lung cancer. Med Sci Monit. 2017;23:5620-9.

35. Saalberg Y, Wolff M. VOC breath biomarkers in lung cancer. Clin Chim Acta. 2016;459:5-9.

36. Cao W, Duan Y. Current status of methods and techniques for breath analysis. Crit Rev Anal Chem. 2007;37(1):3-13.

37. John V. Seeley, Seeley SK. Multidimensional gas chromatography: fundamental advances and new applications. Anal Chem 2012;85(2):557-578

38. Jens Dallüge, Jan Beens, Udo A.Th. Brinkman. Comprehensive two-dimensional gas chromatography: a powerful and versatile analytical tool. J Chromatogr A 2003;1000:69-108.

39. Ma H, Li X, Chen J, Wang H, Cheng $\mathrm{T}$, Chen $\mathrm{K}$, et al. Analysis of human breath samples of lung cancer patients and healthy controls with solid-phase microextraction (SPME) and flow-modulated comprehensive two-dimensional gas chromatography (GC X GC). Anal Methods. 2014;6:6841-9.

40. Beccaria M, Bobak C, Maitshotlo B, Mellors TR, Purcaro G, Franchina FA, et al. Exhaled human breath analysis in active pulmonary tuberculosis diagnostics by comprehensive gas chromatography-mass spectrometry and chemometric techniques. J Breath Res. 2019;13:016005.

41. Phillips M, Cataneo RN, Chaturvedi A, Kaplan PD, Libardoni M, Mundada $M$, et al. Detection of an extended human volatome with comprehensive two-dimensional gas chromatography time-of-flight mass spectrometry. PLoS One. 2013;8: e75274.

42. Tangerman A, Winkel EG. The portable gas chromatograph OralChroma ${ }^{\mathrm{TM}}$ : a method of choice to detect oral and extraoral halitosis. J Breath Res. 2008;2(1):017010-5. 
43. Lee J, Zhou M, Zhu H, Nidetz R, Kurabayashi K, Fan X. Fully automated portable comprehensive 2-dimensional gas chromatography device. Anal Chem. 2016;88:10266-74.

44. Sjoding MW, Hofer TP, Co I, Courey A, Cooke CR, Iwashyna TJ. Interobserver reliability of the Berlin ARDS definition and strategies to improve the reliability of ARDS diagnosis. Chest. 2018;153: 361-7.

45. Zhu H, Nidetz R, Zhou M, Lee J, Buggaveeti S, Kurabayashi $\mathrm{K}$, et al. Flow-through microfluidic photoionization detectors for rapid and highly sensitive vapor detection. Lab Chip. 2015;15: 3021-9.
46. Zhou M, Sharma R, Zhu H, Li J, Wang S, Bisco E, Massey J, Pennington A, Sjoding M, Dickson RP, Park P, Hyzy R, Napolitano L, Ward KR, Fan X. Rapid breath analysis for acute respiratory distress syndrome diagnostics using a portable 2dimensional gas chromatography device. bioRxiv. 2019: https:// www.biorxiv.org/content/10.1101/560888v1. Accessed 3 July 2019.

Publisher's note Springer Nature remains neutral with regard to jurisdictional claims in published maps and institutional affiliations. 